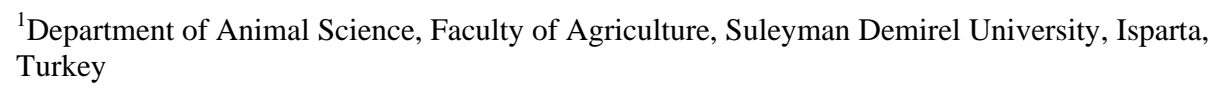

\title{
The relationship of parameters of body measures and body weight by using digital image analysis in pre-slaughter cattle
}

\begin{abstract}
The objective of this study was to predict body weight (BW) of pre-slaughtering beef cattle using digital image analysis. Data used in this study were collected from slaughterhouses in Isparta and nearby provinces from 140 animals. Selected body measurements such as body weight (BW), wither height (WH), body length (BL), chest depth (CD), hip width (HW), hip height $(\mathrm{HH})$ and body area (BA) of different breeds of beef cattle were combined and compared by digital image analysis. The body area was included as a different parameter for prediction of BW instead of chest girth. However, regression equation that included only body area gave the lowest $\mathrm{R}^{2}$ value for Holstein (18.0\%), but the $\mathrm{R}^{2}$ value was 43.2 and $51.7 \%$ for Brown Swiss and crossbred animals, respectively. The regression equation which included all body traits resulted in $\mathrm{R}^{2}$ values 35.3 , 85.1, and 79.6\% for Holstein, Brown Swiss and crossbred, respectively. The regression equation which included body area and body length showed that prediction ability of digital image analysis was high for prediction of BW in Brown Swiss and crossbred animals compared to Holsteins ( $\mathrm{R}^{2} 82.6$, 76.5, and 29.5\%, respectively). Results indicated that the prediction ability of digital image analysis was low for prediction of BW. Although possibility of using body area as a parameter in predicting BW is low it can be developed by further and better designed experiments.
\end{abstract}

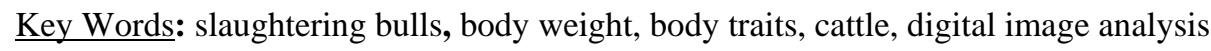

\section{Zusammenfassung}

Titel der Arbeit: Beziehungen von Körpermaßen und Messwerten der digitalen Bildanalyse vor der Schlachtung zum Körpergewicht bei Schlachtrindern

Ziel war die Prüfung einer Vorhersage des Körpergewichtes von Rindern mittels der digitalen Bildanalyse sowie verschiedener Körpermaße. In die Untersuchung waren 56 Holstein Friesian-, 30 Brown Swiss- und 54 Kreuzungsbullen aus Schlachthöfen der Isparta-Provinz einbezogen. Neben dem Körpergewicht wurden die Körpermaße Widerristhöhe, Körperlänge, Hüfthöhe und -breite, Brustumfang und Körperfläche bestimmt. Die einzelnen Messwerte wurden in Beziehung zu den Ergebnissen der digitalen Bildanalyse gesetzt. Die niedrigsten Regressionswerte $\left(\mathrm{R}^{2}\right)$ fanden sich bei Holstein Friesianbullen mit 18 \% während die Werte für Swiss Brown und Kreuzungsbullen bei 43,2 bzw. 51,7 \% lagen. Die $\mathrm{R}^{2}$ Werte welche alle Körpermaße einschlossen betrugen in der genannten Reihenfolge der genetischen Gruppen 35,3, 85,1 bzw. 79,6 \%. $\mathrm{R}^{2}$ Werte welche Körperfläche und Körperlänge einbezogen ergaben für die Brown Swiss und Kreuzungsbullen bessere Werte $\left(\mathrm{R}^{2}=82,6\right.$ bzw. $76,5 \%)$ als für die Holstein Friesian Bullen $\left(\mathrm{R}^{2}=29,5 \%\right)$ Die Ergebnisse zeigten, dass eine Vorhersagemöglichkeit des Körpergewichtes mittels der digitalen Bildanalyse bei der vorliegenden Versuchsanstellung als relativ gering zu beurteilen ist.

Schlüsselworte: Schlachtbullen, Körpergewicht, Körpermaße, digitale Bildanalyse

\section{Introduction}

Body weights (BW) represent one of the most important economic traits in beef cattle. BW is also good indicator of animal condition (POLGAR et al., 1997; VAN MARLEKÖSTER et al., 2000). Methods to estimate weight can be important where weighing facilities are unavailable (ULUTAS et al,. 2002). The use of body weight 
criteria in ration formulation, drug estimation, body condition score, the decision of the date of the first insemination of heifers and marketing requires sophisticated facilities which are expensive and hardly affordable to many small scale farmers (BOZKURT, 2006; WILLEKE and DÜRSCH, 2002).

Several technologies have evaluated to determine the accuracy and precision for predicting body composition. In this purpose, the ultrasound technology began for determine body composition in 1950s (RÖSLER, ZALESKY and BEAL, 2005). McDONALD and CHEN (1990) used digital image analysis technology regarding meat quality investigation in beef cattle that described differences in reflection between meat and fat in Musculus longissimus dorsi (MLD). GERRARD et al., (1996) studied description of colour and degree of marbling on beef. LI et al., (1997) showed that brittleness of meat could be determined by vision texture analysis. Similarly, investigations have shown this method can be used to analyze meat, determine marbling score and evaluate MLD area (NEWMAN, 1984; KUCHIDA et al., 1991; SHACKELFORD et al., 1998; SHIRANITA et al., 2000; KARNUAH et al., 2001; CANNELL et al., 2002; TEIRA et al., 2003). BOZKURT et al., (2006) used digital image analysis technology regarding prediction of body weight from body measurements in beef cattle. Therefore, the objective of this study was to examine prediction of body weight from body measurements and body area (BA) of pre slaughtering beef cattle using digital image analysis. Body measurements obtained by digital image analysis system included body area as a different parameter for prediction of body weight as well as body length, wither height, hip width, hip height and chest depth.

\section{Animal}

\section{Materials and methods}

The animals used in this study included 140 cattle, 56 Holstein, 30 Brown Swiss and 54 crossbred steers. Animals were selected from commercial slaughterhouses in Isparta and nearby provinces. Animals were weighed using a mobile scale before slaughter (Marmara0580 MEB). All procedures were applied by the Institutional Animal care and use committee.

\section{Body Measurements}

Body measurements were taken while animals were standing in a squeeze chute after weighing. Body traits were measured by measuring stick except chest girth, which was measured by measure tape.

Wither Height (WH) - was distance from the ground beneath the animal to the top of the withers directly above the centre of shoulder,

Body Length (BL) - was the distance from point of shoulders to the ischium; in other words, from sternum (manubrium) to the aitchbone (tiber ischiadicum),

Hip Width (HW) - was the widest point at the centre of stifle,

Hip Height $(\mathrm{HH})$ - was distance from the ground beneath the animal to the top of the hips directly above the centre of hip,

Chest Depth (CD) - from sternum area immediately caudal to the forelimbs to top of the thoracic vertebra,

Chest Girth (CG) - was measured as the minimal circumference around the body immediately behind the front shoulder, 
Body Area (BA) - was measure the area covered by animal in digital photo.

The video recording took place outside the slaughterhouse immediately after weighing while animals were standing in a squeeze chute. The video camera (Canon MV850i) was situated of the level of the animals. At the beginning of the analysis of digital images, calibration was conducted using reference card $(1 \times 15 \mathrm{~cm})$. Digital images were downloaded from the camera to a computer file and processed using Image Pro Plus5 software to obtained body measurements from the image in $\mathrm{cm}$.

Statistical Procedure

The best prediction equations for body weight from other body traits, including $\mathrm{WH}$, $\mathrm{BL}, \mathrm{HW}, \mathrm{HH}, \mathrm{CD}$ and BA, were determined. Descriptive statistics and regression analysis of body weight (BW) on each of the independent variables were performed using regression analysis procedure of MINITAB, 13 Inc. (Version 13, State Collage, PA, USA 2001).

Regressions of body weight on WH, BL, HW, CD and CG utilizing individual observations were performed. The body measurements obtained by digital image analysis included BA as a different parameter for prediction of BW instead of CG.

Correlation coefficients were also obtained among parameters. Polynomial regression analysis of body weight on WH, BL, HW, HH, CD and BA were performed.

Linear, quadratic and cubic effects of independent variables on BW were included in the following model:

$Y_{i}=b_{0}+b_{1} X_{i}+b_{2} X_{i}^{2}+b_{3} X_{i}^{3}+e_{i}$

Where $Y_{i}=\mathrm{BW}$ observation of an $i$ th animal, $b_{0}=$ intercept, $b_{1}, b_{2}, b_{3}=$ corresponding linear, quadratic and cubic regression coefficients, $i X=$ independent variables $(\mathrm{WH}$, $\mathrm{BL}, \mathrm{HW}, \mathrm{HH}, \mathrm{BD}$ and BA), $i e=$ residual error term.

\section{Results and discussions}

Descriptive statistics of body weight and body traits are shown in Table 1

Regressions of each breed's body weight on various body measurements using digital image analysis are shown Table 2, 3 and 4. Results of each breed's body weight on linear, quadratic and cubic effects of each body measurement are presented in Table 5. Correlation coefficients of traits are shown in Table 6.

Although all $\mathrm{R}^{2}$ values were found low, the $\mathrm{R}^{2}$ values from regressions indicated that body area was the lowest related to body weight for Holstein using digital image analysis (Table 2) 
Table 1

Descriptive statistic of body weight and body traits (Beschreibende Statistik für Körpergewichte und Körpermaße)

\begin{tabular}{|c|c|c|c|}
\hline Variables & Breeds & $\mathrm{N}$ & Mean \pm SE \\
\hline \multirow[t]{3}{*}{ Body Weight $(\mathrm{cm})$} & Brown Swiss & 30 & $440.7^{\mathrm{a}} \pm 25.7$ \\
\hline & Holstein & 56 & $513.4^{b} \pm 11.6$ \\
\hline & Crossbred & 54 & $460.9^{\mathrm{a}} \pm 13.6$ \\
\hline \multirow[t]{3}{*}{ Body Length (cm) } & Brown Swiss & 30 & $136.88^{\mathrm{a}} \pm 1.97$ \\
\hline & Holstein & 56 & $146.37^{b} \pm 0.95$ \\
\hline & Crossbred & 54 & $140.15^{\mathrm{a}} \pm 1.26$ \\
\hline \multirow[t]{3}{*}{ Wither High (cm) } & Brown Swiss & 30 & $123.45^{\mathrm{a}} \pm 1.40$ \\
\hline & Holstein & 56 & $132,60^{\mathrm{b}} \pm 0.66$ \\
\hline & Crossbred & 54 & $127.95^{\mathrm{c}} \pm 1.14$ \\
\hline \multirow[t]{3}{*}{ Hip High (cm) } & Brown Swiss & 30 & $128,87^{\mathrm{a}} \pm 1.46$ \\
\hline & Holstein & 56 & $137,20^{\mathrm{b}} \pm 0.68$ \\
\hline & Crossbred & 54 & $132.16^{\mathrm{a}} \pm 1.12$ \\
\hline \multirow[t]{3}{*}{ Hip Width (cm) } & Brown Swiss & 30 & $43,283^{\mathrm{a}} \pm 0.87$ \\
\hline & Holstein & 56 & $46.152^{\mathrm{b}} \pm 0.58$ \\
\hline & Crossbred & 54 & $43.222^{\mathrm{a}} \pm 0.56$ \\
\hline \multirow[t]{3}{*}{ Chest Depth (cm) } & Brown Swiss & 30 & $64.78^{\mathrm{a}} \pm 1.12$ \\
\hline & Holstein & 56 & $68.488^{\mathrm{b}} \pm 0.75$ \\
\hline & Crossbred & 54 & $65.074^{\mathrm{a}} \pm 0.68$ \\
\hline \multirow[t]{3}{*}{ Chest Girth (cm) } & Brown Swiss & 30 & $180.25^{\mathrm{a}} \pm 3.38$ \\
\hline & Holstein & 56 & $189.36^{\mathrm{b}} \pm 1.73$ \\
\hline & Crossbred & 54 & $181.59^{\mathrm{a}} \pm .66$ \\
\hline \multirow[t]{3}{*}{ Body Area $\left(\mathrm{cm}^{2}\right)$} & Brown Swiss & 30 & $14549^{a} \pm 408$ \\
\hline & Holstein & 56 & $16464^{\mathrm{b}} \pm 22$ \\
\hline & Crossbred & 54 & $15125^{a} \pm 298$ \\
\hline \multirow[t]{3}{*}{ Body Length $(\mathrm{cm})$} & Brown Swiss & 30 & $139.72^{\mathrm{a}} \pm 1.99$ \\
\hline & Holstein & 56 & $150.33^{\mathrm{b}} \pm 1.06$ \\
\hline & Crossbred & 54 & $143.47^{\mathrm{a}} \pm 1.34$ \\
\hline \multirow[t]{3}{*}{ Wither High (cm) } & Brown Swiss & 30 & $126.25^{\mathrm{a}} \pm 1.36$ \\
\hline & Holstein & 56 & $134.38^{\mathrm{b}} \pm 0.68$ \\
\hline & Crossbred & 54 & $129.96^{\mathrm{a}} \pm 1.21$ \\
\hline \multirow[t]{3}{*}{ Hip High (cm) } & Brown Swiss & 30 & $130.39^{\mathrm{a}} \pm 1.20$ \\
\hline & Holstein & 56 & $139.58^{\mathrm{b}} \pm 0.78$ \\
\hline & Crossbred & 54 & $134.26^{\mathrm{a}} \pm 1.19$ \\
\hline \multirow[t]{3}{*}{ Hip Width (cm) } & Brown Swiss & 30 & $45.284^{\mathrm{a}} \pm 0.87$ \\
\hline & Holstein & 56 & $47.517^{\mathrm{b}}+0.45$ \\
\hline & Crossbred & 54 & $45.372^{\mathrm{a}} \pm 0.70$ \\
\hline \multirow[t]{3}{*}{ Chest Depth (cm) } & Brown Swiss & 30 & $68.01^{\mathrm{a}} \pm 1.25$ \\
\hline & Holstein & 56 & $72.109^{\mathrm{b}} \pm 0.71$ \\
\hline & Crossbred & 54 & $67.917^{\mathrm{a}} \pm 0.82$ \\
\hline
\end{tabular}

Table 2

Prediction equations of body weight and linear effects of selected body traits in Holstein cattle by digital image analysis system (Vorhersagegleichungen und lineare Effekte von Körpermaßen und digitaler Bildanalyse bei Holstein Frisian Bullen)

\begin{tabular}{lcccccccc}
\hline \multicolumn{1}{c}{ Prediction equations } & Constant & $\mathrm{BA}\left(\mathrm{cm}^{2}\right)$ & $\mathrm{BL}$ & $\mathrm{WH}$ & $\mathrm{HH}$ & $\mathrm{HW}$ & $\mathrm{CD}$ & $\mathrm{R}^{2} \%$ \\
\hline $\mathrm{Y}=\mathrm{a}+\mathrm{b}_{1} \mathrm{x}_{1}+\mathrm{b}_{2} \mathrm{x}_{2}+\mathrm{b}_{3} \mathrm{x}_{3}+\mathrm{b}_{4} \mathrm{x}_{4}+\mathrm{b}_{5} \mathrm{x}_{5}+\mathrm{b}_{6} \mathrm{x}_{6}$ & -469 & 0.00159 & 3.27 & 2.22 & -1.68 & 7.12 & 0.86 & 35.3 \\
$\mathrm{Y}=\mathrm{a}+\mathrm{b}_{1} \mathrm{x}_{1}+\mathrm{b}_{2} \mathrm{x}_{2}+\mathrm{b}_{3} \mathrm{x}_{3}+\mathrm{b}_{4} \mathrm{x}_{4}+\mathrm{b}_{5} \mathrm{x}_{5}$ & -485 & 0.00127 & 3.73 & 2.23 & -1.62 & 7.20 & - & 35.2 \\
$\mathrm{Y}=\mathrm{a}+\mathrm{b}_{1} \mathrm{x}_{1}+\mathrm{b}_{2} \mathrm{x}_{2}+\mathrm{b}_{3} \mathrm{x}_{3}+\mathrm{b}_{4} \mathrm{x}_{4}+\mathrm{b}_{6} \mathrm{x}_{6}$ & -531 & 0.00409 & 2.96 & 3.76 & -0.50 & - & 1.32 & 31.5 \\
$\mathrm{Y}=\mathrm{a}+\mathrm{b}_{1} \mathrm{x}_{1}+\mathrm{b}_{2} \mathrm{x}_{2}$ & -333 & 0.00640 & $4.93^{*}$ & - & - & - & - & 29.5 \\
$\mathrm{Y}=\mathrm{a}+\mathrm{b}_{1} \mathrm{x}_{1}+\mathrm{b}_{3} \mathrm{x}_{3}$ & -531 & 0.00822 & - & $6.77^{*}$ & - & - & - & 27.0 \\
$\mathrm{Y}=\mathrm{a}+\mathrm{b}_{1} \mathrm{x}_{1}+\mathrm{b}_{4} \mathrm{x}_{4}$ & -257 & 0.0109 & - & - & 4.24 & - & - & 21.7 \\
$\mathrm{Y}=\mathrm{a}+\mathrm{b}_{1} \mathrm{x}_{1}+\mathrm{b}_{5} \mathrm{x}_{5}$ & -119 & 0.0103 & - & - & - & $9.74^{*}$ & - & 27.5 \\
$\mathrm{Y}=\mathrm{a}+\mathrm{b}_{1} \mathrm{x}_{1}+\mathrm{b}_{6} \mathrm{x}_{6}$ & -96 & 0.0127 & - & - & - & - & 5.55 & 26.5 \\
& & & & & & - & - & 18.0 \\
$\mathrm{Y}=\mathrm{a}+\mathrm{b}_{1} \mathrm{x}_{1}$ & 153 & $0.0219^{*}$ & - & - & - & - & - & 28.6 \\
$\mathrm{Y}=\mathrm{a}+\mathrm{b}_{2} \mathrm{x}_{2}$ & -363 & - & $5.83^{*}$ & - & - & - & - & 25.5 \\
$\mathrm{Y}=\mathrm{a}+\mathrm{b}_{3} \mathrm{x}_{3}$ & -637 & - & - & $8.56^{*}$ & - & - & - & - \\
$\mathrm{Y}=\mathrm{a}+\mathrm{b}_{4} \mathrm{x}_{4}$ & -401 & - & - & - & $6.55^{*}$ & - & - & 19.7 \\
$\mathrm{Y}=\mathrm{a}+\mathrm{b}_{5} \mathrm{x}_{5}$ & -93 & - & - & - & - & $12.8^{*}$ & - & 24.9 \\
$\mathrm{Y}=\mathrm{a}+\mathrm{b}_{6} \mathrm{x}_{6}$ & -38 & - & - & - & - & - & 7.64 & 22.1 \\
& & & & & & & $*$
\end{tabular}


Inclusion of $\mathrm{BL}$ in the equation increased $\mathrm{R}^{2}$ greatly in every steps of regression for both Brown Swiss and crossbred (Table 3 and 4 ). The $\mathrm{R}^{2}$ values of body area with digital image analysis for Brown Swiss and crossbred animals were 43.2 and 51.7, respectively. However the regression equations included both body area and body length showed the highest $\mathrm{R}^{2}$ for Brown Swiss and crossbred animals $\left(\mathrm{R}^{2}=82.6\right.$ and 79.6, respectively). The equation included all body traits except CD or HW showed the highest $\mathrm{R}^{2}$ for Brown Swiss $\left(\mathrm{R}^{2}=85.0 \%\right)$. However, the equation that included all body traits except $\mathrm{HH}$ was $\mathrm{R}^{2}=79.4 \%$ for crossbred. The $\mathrm{R}^{2}$ values which obtained from equation contained BA and BL were found to be $82.6 \%$ and $76.5 \%$ for Brown Swiss and crossbred respectively. The prediction equation including both BA and BL analyzed by digital image analysis resulted in better prediction than the rest of the equations contained other body traits. The results indicated that BL can be used to predict body weight accurately for Brown Swiss and crossbred cattle $\left(\mathrm{R}^{2}=80.7 \%\right.$ and $75.6 \%$, respectively) (Table 3). These results were higher with findings of BOZKURT (2006) and ULUTAS et al., (2002) who reported that the $\mathrm{R}^{2}$ values for BL was $70.4 \%$ and 68.3\%, respectively with traditional method. BOZKURT et al., (2006) reported that $\mathrm{R}^{2}$ value for $\mathrm{BL}$ was $63.6 \%$ with digital image analysis. HEINRICHS et al., (1992) and SEKERDEN et al., (1991) indicated that BL can be used to estimate BW from body weight $\left(\mathrm{R}^{2}=93 \%\right.$ and $96.1 \%$, respectively). The relationship between BA and change in body weight is presented in Table 3,4 . The results showed that a $1 \mathrm{~cm}^{2}$ change in BA resulted in approximately 0.041 and $0.0328 \mathrm{~kg}$ change in weigh for Brown Swiss and crossbred cattle, respectively. It was evident that a $1 \mathrm{~cm}^{2}$ change in BA resulted in lesser weight change compared to the rest of body traits (Table 3 and 4). Similarly, a $1 \mathrm{~cm}$ change BL, WH, HH, HW and CD resulted in 11.6, 14.8, 15.3, 22.4 and $17.6 \mathrm{~kg}$ change in weight for Brown Swiss and 8.80, 8.97, 9.05, 12.7 and $11.6 \mathrm{~kg}$ change for crossbred, respectively.

Table 3

Prediction equations of body weight and linear effects of selected body traits in Brown Swiss cattle by digital image analysis system (Vorhersagegleichungen und lineare Effekte von Körpermaßen und digitaler Bildanalyse bei Brown Swiss Bullen)

\begin{tabular}{|c|c|c|c|c|c|c|c|c|}
\hline Prediction equations & Constant & $\mathrm{BA}\left(\mathrm{cm}^{2}\right)$ & BL & WH & $\mathrm{HH}$ & HW & CD & $\mathrm{R}^{2} \%$ \\
\hline $\mathrm{Y}=\mathrm{a}+\mathrm{b}_{1} \mathrm{x}_{1}+\mathrm{b}_{2} \mathrm{x}_{2}+\mathrm{b}_{3} \mathrm{x}_{3}+\mathrm{b}_{4} \mathrm{x}_{4}+\mathrm{b}_{5} \mathrm{x}_{5}+\mathrm{b}_{6} \mathrm{x}_{6}$ & -1866 & $-0.0266^{*}$ & $12.8^{*}$ & 1.52 & 5.72 & -1.39 & 0.46 & 85.1 \\
\hline $\mathrm{Y}=\mathrm{a}+\mathrm{b}_{1} \mathrm{x}_{1}+\mathrm{b}_{2} \mathrm{x}_{2}+\mathrm{b}_{3} \mathrm{x}_{3}+\mathrm{b}_{4} \mathrm{x}_{4}+\mathrm{b}_{5} \mathrm{x}_{5}$ & -1880 & $-0.0267^{*}$ & $13.0^{*}$ & 1.57 & 5.78 & -1.41 & - & 85.0 \\
\hline $\mathrm{Y}=\mathrm{a}+\mathrm{b}_{1} \mathrm{x}_{1}+\mathrm{b}_{2} \mathrm{x}_{2}+\mathrm{b}_{3} \mathrm{x}_{3}+\mathrm{b}_{4} \mathrm{x}_{4}+\mathrm{b}_{6} \mathrm{x}_{6}$ & -1844 & $-0.0261^{*}$ & $12.2^{*}$ & 1.67 & 5.47 & - & 0.51 & 85.0 \\
\hline $\mathrm{Y}=\mathrm{a}+\mathrm{b}_{1} \mathrm{x}_{1}+\mathrm{b}_{2} \mathrm{x}_{2}$ & -1315 & -0.0150 & $14.1^{*}$ & - & - & - & - & 82.6 \\
\hline $\mathrm{Y}=\mathrm{a}+\mathrm{b}_{1} \mathrm{x}_{1}+\mathrm{b}_{3} \mathrm{x}_{3}$ & -1286 & 0.0103 & - & $12.5^{*}$ & - & - & - & 62.8 \\
\hline $\mathrm{Y}=\mathrm{a}+\mathrm{b}_{1} \mathrm{x}_{1+} \mathrm{b}_{4} \mathrm{x}_{4}$ & -1284 & 0.0122 & - & - & $11.9^{*}$ & - & - & 52.5 \\
\hline $\mathrm{Y}=\mathrm{a}+\mathrm{b}_{1} \mathrm{x}_{1}+\mathrm{b}_{5} \mathrm{x}_{5}$ & -572 & 0.0166 & - & - & - & $17.0^{*}$ & - & 60.8 \\
\hline $\mathrm{Y}=\mathrm{a}+\mathrm{b}_{1} \mathrm{x}_{1}+\mathrm{b}_{6} \mathrm{x}_{6}$ & -752 & 0.00072 & - & - & - & - & $17.4^{*}$ & 72.9 \\
\hline $\mathrm{Y}=\mathrm{a}+\mathrm{b}_{1} \mathrm{x}_{1}$ & -161 & $0.0414^{*}$ & - & - & - & - & - & 43.2 \\
\hline $\mathrm{Y}=\mathrm{a}+\mathrm{b}_{2} \mathrm{x}_{2}$ & -1181 & - & $11.6^{*}$ & - & - & - & - & 80.7 \\
\hline $\mathrm{Y}=\mathrm{a}+\mathrm{b}_{3} \mathrm{x}_{3}$ & -1425 & - & - & $14.8^{*}$ & - & - & - & 61.6 \\
\hline $\mathrm{Y}=\mathrm{a}+\mathrm{b}_{4} \mathrm{x}_{4}$ & -1558 & - & - & - & $15.3^{*}$ & - & - & 51.3 \\
\hline $\mathrm{Y}=\mathrm{a}+\mathrm{b}_{1} \mathrm{x}_{1}$ & -572 & - & - & - & - & $22.4^{*}$ & - & 57.1 \\
\hline $\mathrm{Y}=\mathrm{a}+\mathrm{b}_{2} \mathrm{X}_{2}$ & -754 & - & - & - & - & - & $17.6^{*}$ & 72.9 \\
\hline
\end{tabular}

* Statistically significant $(\mathrm{P}<0.05) \mathrm{b}$ is coefficient of variables, $\mathrm{X}$ is independent variables $(\mathrm{WH}, \mathrm{BL}, \mathrm{HW}, \mathrm{HH}, \mathrm{BD}$ and $\mathrm{BA})$ 
Table 4

Prediction equations of body weight and linear effects of selected body traits in crossbred cattle by digital image analysis system (Vorhersagegleichungen und lineare Effekte von Körpermaßen und digitaler Bildanalyse bei Kreuzungsbullen).

\begin{tabular}{|c|c|c|c|c|c|c|c|c|}
\hline Prediction equations & Constant & $\mathrm{BA}\left(\mathrm{cm}^{2}\right)$ & BL & WH & $\mathrm{HH}$ & HW & $\mathrm{CD}$ & $\overline{\mathrm{R}^{2} \%}$ \\
\hline $\mathrm{Y}=\mathrm{a}+\mathrm{b}_{1} \mathrm{x}_{1}+\mathrm{b}_{2} \mathrm{x}_{2}+\mathrm{b}_{3} \mathrm{x}_{3}+\mathrm{b}_{4} \mathrm{x}_{4}+\mathrm{b}_{5} \mathrm{x}_{5}+\mathrm{b}_{6} \mathrm{x}_{6}$ & -767 & 0.00699 & $7.85^{*}$ & 2.20 & -1.54 & 3.24 & -3.41 & 79.6 \\
\hline $\mathrm{Y}=\mathrm{a}+\mathrm{b}_{1} \mathrm{x}_{1}+\mathrm{b}_{2} \mathrm{x}_{2}+\mathrm{b}_{3} \mathrm{x}_{3}+\mathrm{b}_{4} \mathrm{x}_{4}+\mathrm{b}_{5} \mathrm{x}_{5}$ & -742 & 0.00662 & $6.10^{*}$ & 1.66 & -1.06 & 3.38 & - & 78.6 \\
\hline $\mathrm{Y}=\mathrm{a}+\mathrm{b}_{1} \mathrm{x}_{1}+\mathrm{b}_{2} \mathrm{x}_{2}+\mathrm{b}_{3} \mathrm{x}_{3}+\mathrm{b}_{4} \mathrm{x}_{4}+\mathrm{b}_{6} \mathrm{x}_{6}$ & -787 & 0.00655 & $8.81^{*}$ & 2.14 & -1.08 & - & -3.65 & 78.0 \\
\hline $\mathrm{Y}=\mathrm{a}+\mathrm{b}_{1} \mathrm{x}_{1}+\mathrm{b}_{2} \mathrm{X}_{2}$ & -743 & 0.00659 & $7.70^{*}$ & - & - & - & - & 76.5 \\
\hline$Y=a+b_{1} x_{1}+b_{3} x_{3}$ & -595 & $0.0134^{*}$ & - & $6.57^{*}$ & - & - & - & 67.8 \\
\hline $\mathrm{Y}=\mathrm{a}+\mathrm{b}_{1} \mathrm{x}_{1+} \mathrm{b}_{4} \mathrm{x}_{4}$ & -626 & $0.0121^{*}$ & - & - & $6.73^{*}$ & - & - & 66.2 \\
\hline $\mathrm{Y}=\mathrm{a}+\mathrm{b}_{1} \mathrm{x}_{1}+\mathrm{b}_{5} \mathrm{x}_{5}$ & -259 & $0.0241^{*}$ & - & - & - & $7.85^{*}$ & - & 64.3 \\
\hline $\mathrm{Y}=\mathrm{a}+\mathrm{b}_{1} \mathrm{x}_{1}+\mathrm{b}_{6} \mathrm{x}_{6}$ & -304 & $0.0203^{*}$ & - & - & - & - & $6.74^{*}$ & 61.0 \\
\hline $\mathrm{Y}=\mathrm{a}+\mathrm{b}_{1} \mathrm{x}_{1}$ & -34.5 & $0.0328^{*}$ & - & - & - & - & - & 51.7 \\
\hline $\mathrm{Y}=\mathrm{a}+\mathrm{b}_{2} \mathrm{X}_{2}$ & -802 & - & $8.80^{*}$ & - & - & - & - & 75.6 \\
\hline $\mathrm{Y}=\mathrm{a}+\mathrm{b}_{3} \mathrm{X}_{3}$ & -705 & - & - & $8.97^{*}$ & - & - & - & 63.7 \\
\hline $\mathrm{Y}=\mathrm{a}+\mathrm{b}_{4} \mathrm{x}_{4}$ & -754 & - & - & - & $9.05^{*}$ & - & - & 63.3 \\
\hline $\mathrm{Y}=\mathrm{a}+\mathrm{b}_{5} \mathrm{x}_{5}$ & -116 & - & - & - & - & $12.7^{*}$ & - & 42.7 \\
\hline $\mathrm{Y}=\mathrm{a}+\mathrm{b}_{6} \mathrm{x}_{6}$ & -330 & - & - & - & - & - & $11.6^{*}$ & 50.0 \\
\hline
\end{tabular}

* Statistically significant $(\mathrm{P}<0.05) \mathrm{b}$ is coefficient of variables, $\mathrm{X}$ is independent variables $(\mathrm{WH}, \mathrm{BL}, \mathrm{HW}, \mathrm{HH}, \mathrm{BD}$ and $\mathrm{BA})$

Higher order polynomial equations were examined (Table 5). The $\mathrm{R}^{2}$ values from regressions indicate that BL was highly related to body weight considering all linear, quadratic and cubic coefficient terms for all breeds. The cubic term was non significant ( $>$ >0.05) for all traits except WH in Brown Swiss (Table 5). HEINRICHS et al., (1992) reported that the cubic term was significant for WH, HW and BL. For all body traits, addition of cubic term increased the $\mathrm{R}^{2}$ slightly for all breeds (Table 5). All linear terms for all body traits of all breeds were significant $(\mathrm{P}<0.05)$.

All correlation values between body weight and body measurements for Holstein, Brown Swiss and crossbred animals were found to be statistically significant $(\mathrm{P}<$ 0.05). Among all body measurements, high correlation was found between body weight and body length for all breed except Holstein (Table 6). The correlation was found 0.54, 0.89 and 0.87 between body weight and body length in Holstein, Brown Swiss and Crossbred, respectively and this result was in line with findings of BOZKURT (2006) who reported that $\mathrm{r}=0.84$ in Brown Swiss. TOZSER et al. (2000); CAGLAR and SEKERDEN (1993) and SEKERDEN et al., (1991) represented that correlation coefficient between BW and BL was $0.63,0.74$ and 0.98 respectively. The correlation between BW and BA was found 0.43, 0.66 and 0.72 for Holstein, Brown Swiss and Crossbred, respectively. It was expected that body area would give higher correlation coefficient value than the other body measurements since the $\mathrm{R}^{2}$ value between body weight and body area was not high. 
Table 5

Regressions of body weight on the linear, quadratic and cubic effects of each body measurement ${ }^{\#}$ (Regressionen des

Körpergewichts auf lineare, quadratische und kubische Effekte bei den einzelnen Merkmalen)

\begin{tabular}{|c|c|c|c|c|c|}
\hline Measurements & Constant & Linear & Quadratic & Cubic & $\mathbf{R}^{2} \%$ \\
\hline \multicolumn{6}{|l|}{ Holstein } \\
\hline \multirow[t]{3}{*}{ Body Area } & 153.3 & 0.02 & - & - & 18.0 \\
\hline & 422.2 & -0.01 & $0.00^{\mathrm{ns}}$ & - & 18.2 \\
\hline & -4007.7 & -0.82 & $-0.000^{\mathrm{ns}}$ & $0.00^{\mathrm{ns}}$ & 19.2 \\
\hline \multirow[t]{3}{*}{ Body Length } & -362.7 & 5.83 & - & - & 28.6 \\
\hline & -2723.7 & 36.77 & $-0.10^{\mathrm{ns}}$ & - & 29.8 \\
\hline & -24565.5 & 466.97 & $-2.92^{\mathrm{ns}}$ & $0.01^{\mathrm{ns}}$ & 30.5 \\
\hline \multirow[t]{3}{*}{ Wither High } & -636.9 & 8.56 & - & - & 25.5 \\
\hline & -3201.2 & 46.94 & $-0.14^{\mathrm{ns}}$ & - & 25.9 \\
\hline & -66446.1 & 1475.34 & $-10.88^{\mathrm{ns}}$ & $0.03^{\mathrm{ns}}$ & 26.4 \\
\hline \multirow[t]{3}{*}{ Hip High } & -401.4 & 6.55 & - & - & 19.7 \\
\hline & -4038.5 & 58.30 & $-0.18^{\text {ns }}$ & - & 20.4 \\
\hline & -38967.6 & 803.70 & $-5.48^{\mathrm{ns}}$ & $0.01^{\mathrm{ns}}$ & 20.5 \\
\hline \multirow[t]{3}{*}{ Hip Width } & -93.1 & 12.76 & - & - & 24.9 \\
\hline & -1933.7 & 91.54 & $0.84^{\mathrm{ns}}$ & - & 27.2 \\
\hline & 38701.8 & -2554.17 & $56.27^{\mathrm{ns}}$ & -0.41 & 37.4 \\
\hline \multirow[t]{3}{*}{ Chest Depth } & -37.7 & 7.34 & - & - & 22.1 \\
\hline & 132.5 & 2.93 & $0.03^{\mathrm{ns}}$ & - & 22.1 \\
\hline & -17444.1 & 745.92 & $-10.37^{\mathrm{ns}}$ & $0.05^{\mathrm{ns}}$ & 25.5 \\
\hline \multicolumn{6}{|l|}{ Brown Swiss } \\
\hline \multirow[t]{3}{*}{ Body Area } & -161.0 & 0.04 & - & - & 43.2 \\
\hline & 1372.5 & -0.16 & $0.00^{\mathrm{ns}}$ & - & 48.5 \\
\hline & 10833.9 & -1.99 & $0.00^{\mathrm{ns}}$ & $-0.00^{\mathrm{ns}}$ & 51.3 \\
\hline \multirow[t]{3}{*}{ Body Length } & -1180.9 & 11.61 & - & - & 80.7 \\
\hline & 1918.5 & -30.61 & $0.14^{\mathrm{ns}}$ & - & 82.4 \\
\hline & -16191.6 & 343.27 & $-2.42^{\mathrm{ns}}$ & $0.01^{\mathrm{ns}}$ & 82.7 \\
\hline \multirow[t]{3}{*}{ Wither High } & -1425.3 & 14.78 & - & - & 61.6 \\
\hline & 4850.4 & -82.11 & $0.37^{\mathrm{ns}}$ & - & 65.2 \\
\hline & 127149 & -2940.93 & $22.58^{\mathrm{ns}}$ & -0.06 & 72.6 \\
\hline \multirow[t]{3}{*}{ Hip High } & -1557.8 & 15.33 & - & - & 51.3 \\
\hline & 8920.8 & -142.55 & $0.59^{\mathrm{ns}}$ & - & 55.4 \\
\hline & 132897 & -2941.55 & $21.62^{\mathrm{ns}}$ & $-0.05^{\mathrm{ns}}$ & 57.0 \\
\hline \multirow[t]{3}{*}{ Hip Width } & -572.2 & 22.37 & - & - & 57.1 \\
\hline & 4635.5 & -197.49 & 2.29 & - & 74.1 \\
\hline & -10763.2 & 775.16 & -18.03 & $0.14^{\mathrm{ns}}$ & 75.6 \\
\hline \multirow[t]{3}{*}{ Chest Depth } & -754.1 & 17.57 & - & - & 72.9 \\
\hline & 2742.6 & -79.71 & 0.67 & - & 78.0 \\
\hline & -18281.9 & 808.07 & -11.74 & $0.06^{\mathrm{ns}}$ & 79.5 \\
\hline \multicolumn{6}{|l|}{ Crossbred } \\
\hline \multirow[t]{3}{*}{ Body Area } & -34.5 & 0.03 & - & - & 51.7 \\
\hline & -638.9 & 0.11 & $-0.000^{\mathrm{ns}}$ & - & 53.9 \\
\hline & 1878.8 & -0.36 & $0.000^{\mathrm{ns}}$ & $-0.00^{\mathrm{ns}}$ & 54.9 \\
\hline \multirow[t]{3}{*}{ Body Length } & -802.3 & 8.80 & - & - & 75.6 \\
\hline & 1145.8 & -18.38 & $0.09^{\mathrm{ns}}$ & - & 76.7 \\
\hline & 48047.8 & -1000.16 & $6.92^{\mathrm{ns}}$ & -0.02 & 80.1 \\
\hline Wither High & -704.9 & 8.97 & - & - & 63.7 \\
\hline & -727.2 & 9.31 & $-0.00^{\mathrm{ns}}$ & - & 63.7 \\
\hline & 35457.2 & -819.74 & $6.31^{\mathrm{ns}}$ & $-0.02^{\mathrm{ns}}$ & 65.8 \\
\hline Hip High & -753.7 & 9.04 & - & - & 63.3 \\
\hline & -615.9 & 7.01 & $0.01^{\mathrm{ns}}$ & - & 63.3 \\
\hline & 33750.3 & -754.96 & $5.62^{\mathrm{ns}}$ & $-0.01^{\mathrm{ns}}$ & 64.4 \\
\hline Hip Width & -115.8 & 12.71 & - & - & 42.7 \\
\hline & -1534.2 & 74.08 & -0.65 & - & 51.7 \\
\hline & 9177.57 & -608.58 & 13.62 & -0.09 & 65.1 \\
\hline Chest Depth & -329.9 & 11.64 & - & - & 50.0 \\
\hline & 319.0 & -7.35 & $0.14^{\mathrm{ns}}$ & - & 50.4 \\
\hline & 10246.1 & -452.23 & $6.73^{\mathrm{ns}}$ & $-0.03^{\text {ns }}$ & 51.5 \\
\hline
\end{tabular}

\# Only none significant regression coefficients had superscripts (ns), the rest were significant at $\mathrm{p}<0.05$. 
Pearson correlations between body weight and selected body traits in breeds (Pearson Korrelationskoeffizienten zwischen Körpergewicht und den einzelnen Messmerkmalen)

\begin{tabular}{cccc}
\hline Variables & Holstein & $\begin{array}{c}\text { Body Weight } \\
\text { Brown Swiss }\end{array}$ & Crossbred \\
\hline Body Area & 0.43 & 0.66 & 0.72 \\
Body Length & 0.54 & 0.89 & 0.87 \\
Wither High & 0.51 & 0.79 & 0.80 \\
Hip High & 0.44 & 0.72 & 0.80 \\
Hip Width & 0.50 & 0.76 & 0.65 \\
Chest Depth & 0.47 & 0.85 & 0.71 \\
\hline
\end{tabular}

It could be concluded that the digital image analysis can predict the body weight from body traits. When the animals have large frame size such as Holstein prediction of body weight, was lower than prediction obtained in Brown Swiss and Crossbred cattle. However, BA and BL obtained by digital image analysis may be better parameters in predicting weight of Brown Swiss and crossbred cattle.

The digital image analysis is promising approach in predicting body weight of live animals. However, in order to increase accuracy, better controlled conditions should be provided wherein cattle are fixed in squeeze chute. Digital image analysis can be developed to prediction body weight by further and better designed experiments.

\section{Acknowledgments}

This project was funded by Suleyman Demirel University Research Council and Evren Meat Packing Company. We also thank Dr. Sedat Aktan, Dr. Birol Kilic, Tolga Hancer, Begum Ozbek, Turgay Akunal, Hasbi Duman, Orhan Askin for their contributions.

BOZKURT, Y.:

\section{References}

Prediction of body weight from body size measurements in Brown Swiss feedlot cattle fed under smallscale farming conditions. J. of Appl. Anim. Res. 29 (2006) 1, 29-32

BOZKURT, Y.; AKTAN, S.; OZKAYA, S.:

Body weight prediction using digital image analysis for slaughtering beef cattle. EAAP, Antalya, Turkey.17-20 September, 2006. Abstract No. 12, Session C33 No.13 Page: 313

CAGLAR, H.; SEKERDEN, O.:

Predicting live weight from various body measurements in Yerlikara cattle. J. of Agric. Collage of Ondokuzmayis Unviersity 8 (1993) 1, 45-47

CANNELL, R.C.; BELK, K.E.; TATUM, J.D.; WISE, J.W.; CHAPMAN, P.L.; SCANGA, J.A.; SMITH, G.C.: Online evaluation of a commercial video image analysis system (Computer vision system) to predict beef carcass red meat yield and for augmenting the assignment of USDA yield grades. J. of Anim. Sci. 80 (2002), 1195-1201

GERRARD, D.E.; GEO, X; TAN, J.:

Beef marbling and colour score determination by image processing. J. of Food Sci. 61 (1996) 1, 145148

HEINRICHS, A.J.; ROGERS, G.W.; COOPER, J.B.:

Predicting body weight and wither height in Holstein heifers using body measurements. J. of Dairy Sci. 75 (1992), 3576-3581

KARNUAH, A.B.; MORIYA, K.; NAKANISHI, N.; NADE, T.; MITSUHASHI, T.; SASAKI, Y.:

Computer image analysis for prediction of carcass composition from carcass-sections of Japanese Black steers. J. of Anim. Sci. 79 (2001), 2851-2856

KUCHIDA, K.; SUZUKI, K.; YAMAKI, K.; SHIROHARA, H.; YAMAGISHI, T.:

Prediction for chemical component of pork meat by personal computer color image analysis. Anim. Sci. Techn. 62 (1991), 477-479

LI, J.; TAN, J.; MARTZ, F.A.:

Predicting beef tenderness from image texture features. 1997 ASAE annual international meeting technical papers, paper no. 973124, ASAE, 2950 Niles Road, St. Joseph, MI 49085-9659, USA 
MINITAB Institute Inc. MINITAB User’s Guide. Release 13 for Windows. MINITAB. Inc. State Collage, PA, USA. 2001

MCDONALD, T.; CHEN, Y.R.:

Separating connected muscle tissues in image of beef carcass rib eyes. Transactions of the ASAE. 33 (1990) 6, 187-193

NEWMAN, P.B.:

The use of video image analysis for quantitative measurements of fatness in meat: Part 2 Comparison of VIA, visual assessment and chemical fat estimation in a commercial environment. Meat Sci. 10 (1984), 161-166

POLGAR, P.; SZÜCS, E.; SZABO, F.

Effect of selection for improvement of dairy performance on growth and body structure in Holstein Friesian and Hungarian Fleckvieh young bulls. (German language) Arch. Tierz. 40 (1997) 505-510

RÖSLER, H.-J.; LENGERKEN von, G.; WICKE, M.; SCHEEDER, M.R.L.; BEISCH, B. Investigations on the accuracy and the application of ultrasonic measurements for prediction carcass composition on live cattle. (German language) Arch. Tierz. 39 (1996) 404-413

SCHACKELFORD, S.D.; WHEELER, T.L.; KOOHMARAIE, M.:

Coupling if image analysis and tenderness classification to simultaneously evaluate carcass cutability, longissimus area, subprimal cut weights and tenderness of beef. J. of Anim. Sci. 76 (1998), 2631-2640

SEKERDEN, O.; KURAN, M.; SEN, O.S.:

Predicting live weight from various body measurements in Black-White cattle. J. of Vet. and Anim. Sci. 16 (1991), 93-102

SHIRANITA, K.; MIYAJIMA, T.; TAKIYAMA, R.:

Determination of meat quality by texture analysis. Pattern Recognition Leters. 19 (1998), 1319-1324

TEIRA, G.A.; TINOIS, E.; LOTUFO, R. A.; FELICIO, P.E.:

Digital-image analysis to predict weight and yields of boneless subprimal beef cuts. Sci. Agric. 60 (2003) 2, 403-408

TOZSER, J.; DOMOKOS, Z.; RUSZNAK, J.; SZELENYI, L.; TOZSER, G.G.: Data on body measurements of Charolasis cows. 2000. www.atk.hu/English/npaper/summ00-3.htm.

ULUTAS, Z; SAATCI, M.; OZLUTURK, A.:

Prediction of body weights from body measurements in East Anatolian Red calves. Indian J. of Anim. Sci. 72 (2002) 10, 878-881

VAN MARLE-KÖSTER, E.; MOSTERT, B.E.; VAN der WESTHUIZEN, J.

Body measurements as selection criteria for growth in South African Hereford cattle. Arch. Tierz. 43 (2000) 5-15

WILLEKE, H.; DÜRSCH, T.:

Prediction of the body weight of Simmental heifers using heart girth measurements. (German language) Arch. Tierz. 45 (2002) 1, 23-28

ZALESKY, D.; Beal, W.E.:

Using Ultrasound Technology in Beef Cattle Reproductive Management. Beef Cattle Handbook, BCH2400 (2005) p. 1-3

Received: 2007-03-07

Accepted: 2008 01-11

Corresponding Author

SERKAN OZKAYA, Ph.D

Suleyman Demirel University, Agriculture Faculty, Department of Animal Science

32260 Cunur - ISPARTA / TURKIYE

email: sozkaya@ziraat.sdu.edu.tr 